KEK-TH-1111

July 18, 2018

\title{
Non-perturbative Effect on Thermal Relic Abundance of Dark Matter
}

\author{
Junji Hisano $^{(a)}$, Shigeki Matsumoto ${ }^{(b)}$, Minoru Nagai ${ }^{(a)}$, Osamu Saito $^{(a, b)}$, \\ and Masato Senami ${ }^{(a)}$ \\ (a) ICRR, University of Tokyo, Kashiwa 277-8582, Japan \\ (b) Theory Group, KEK, Oho 1-1 Tsukuba, Ibaraki 305-0801, Japan
}

\begin{abstract}
We point out that thermal relic abundance of the dark matter is strongly altered by a non-perturbative effect called the Sommerfeld enhancement, when constituent particles of the dark matter are non-singlet under the $\mathrm{SU}(2)_{L}$ gauge interaction and much heavier than the weak gauge bosons. Typical candidates for such dark matter particles are the heavy wino- and higgsino-like neutralinos. We investigate the non-perturbative effect on the relic abundance of dark matter for the wino-like neutralino as an example. We show that its thermal abundance is reduced by $50 \%$ compared to the perturbative result. The wino-like neutralino mass consistent with the observed dark matter abundance turns out to be $2.7 \mathrm{TeV} \lesssim m \lesssim 3.0 \mathrm{TeV}$.
\end{abstract}




\section{Introduction}

Precise measurements of cosmological parameters have achieved amazing progress in recent years. In particular, the observation of cosmic microwave background anisotropies by the Wilkinson Microwave Anisotropy Probe (WMAP) [1] confirmed that non-baryonic dark matter amounts to $20 \%$ of the energy of our universe. The existence of dark matter forces us to consider physics beyond the standard model (SM) for its constituent, because the SM has no candidate for the dark matter.

Many models beyond the SM have been proposed for providing dark matter candidates. Models involving weakly interacting massive particles (WIMPs), such as supersymmetric models and universal extra dimension (UED) models 2, 3, have an advantage over other models, since the WIMPs can explain the observed abundance naturally in the thermal relic scenario [3, 4, 5]. Comparison of the predicted thermal abundance with observations is a powerful tool to constrain those models. It is now important for discussion of new physics signatures at collider experiments such as the LHC.

In the thermal relic scenario, the annihilation cross sections of dark matter play a central role in evaluation of the abundance. Perturbative calculation of the cross sections is sufficient for it in usual cases. However, a non-perturbative effect on the cross sections has to be included, when dark matter particles are non-singlet under the $\mathrm{SU}(2)_{L}$ interaction and much heavier than the weak gauge bosons. The weak interaction is not a short-distance force, but rather a long-distance one for non-relativistic two-bodies states of such heavy particles. The wave functions for the two-bodes states are modified from the plane waves by the interaction, and the annihilation cross sections are affected. The dark matter particles are non-relativistic at the freeze-out temperature. The attractive channels due to the weak force enhance the annihilation cross sections, compared with the perturbative ones. This effect is called the Sommerfeld enhancement, which was found in inelastic reactions between non-relativistic charged particles, historically.

In this letter, we point out that the non-perturbative effect strongly alters the relic abundance of dark matter compared to that in perturbative calculation. We investigate the non-perturbative effect quantitatively in the wino-like neutralino dark matter as an example, and show that the abundance is reduced by $50 \%$ due to the effect. We also discuss non-perturbative effects on other dark matter candidates at the end of this letter. 


\section{Sommerfeld enhancement in wino-like neutralino dark matter annihilation}

Winos are the $\mathrm{SU}(2)_{L}$ gauginos in the supersymmetric standard model, and they are an $\mathrm{SU}(2)_{L}$ triplet. They are mixed with higgsinos, superpartners of the Higgs bosons, after the electroweak symmetry breaking. The neutral components in the mass eigenstates are called neutralino. The mixing with higgsinos is small for heavy winos, since it is suppressed by the electroweak scale. Thus, the wino-like neutralino $\left(\tilde{\chi}^{0}\right)$ is highly degenerate with its charged $\mathrm{SU}(2)_{L}$ partner $\left(\tilde{\chi}^{-}\right)$in mass when their masses are heavy enough. For the wino-like neutralino mass $(m)$ of the order of $1 \mathrm{TeV}$, the mass difference between the neutralino and its $\mathrm{SU}(2)_{L}$ partner is dominated by a radiative correction [6]. It is calculated as $\delta m \simeq 0.17 \mathrm{GeV}$, which is used for numerical calculations in this letter.

There are four processes related to the calculation of the wino-like neutralino relic abundance, and those are $\tilde{\chi}^{0} \tilde{\chi}^{0}, \tilde{\chi}^{-} \tilde{\chi}^{+}, \tilde{\chi}^{0} \tilde{\chi}^{-}$, and $\tilde{\chi}^{-} \tilde{\chi}^{-}$annihilation. We assume the $\mathrm{CP}$ conservation, and cross sections of $\tilde{\chi}^{0} \tilde{\chi}^{+}$and $\tilde{\chi}^{+} \tilde{\chi}^{+}$annihilation are the same as those of $\tilde{\chi}^{0} \tilde{\chi}^{-}$and $\tilde{\chi}^{-} \tilde{\chi}^{-}$, respectively. Furthermore, each process is decomposed into two ones with $S=0$ and $S=1$, where $S$ is the total spin of the two-bodies system at the initial state. In $\tilde{\chi}^{0} \tilde{\chi}^{0}$ and $\tilde{\chi}^{-} \tilde{\chi}^{-}$annihilation, the $S=1$ processes are forbidden at the $s$-wave annihilation. After all, processes we have to consider are $\tilde{\chi}^{0} \tilde{\chi}^{0}, \tilde{\chi}^{-} \tilde{\chi}^{+}, \tilde{\chi}^{0} \tilde{\chi}^{-}, \tilde{\chi}^{-} \tilde{\chi}^{-}$annihilation with $S=0$, and those of $\tilde{\chi}^{-} \tilde{\chi}^{+}, \tilde{\chi}^{0} \tilde{\chi}^{-}$with $S=1$.

While gamma rays from the wino-like neutralino annihilation in the Galactic center [6, 7] and anti-particles fluxes from that in Galactic Halo [8] are evaluated including non-perturbative effects, the relic abundance of the neutralino have been calculated only within a perturbative method. However, as we will see later, the nonperturbative effect can significantly alter cross sections relevant to the abundance.

Two-bodies effective Lagrangian, describing relative motion between two particles in the two-bodies system, is useful to evaluate the non-perturbative effect in the annihilation. The Lagrangian has a following form,

$$
\mathcal{L}_{\mathrm{eff}}=\int d^{3} r \sum_{S_{Z}} \Phi_{S_{Z}}^{\dagger}(x, \vec{r})\left[i \partial_{x^{0}}+\frac{\nabla_{x}^{2}}{4 m}+\frac{\nabla_{r}^{2}}{m}-V(r)+2 i \Gamma \delta(\vec{r})\right] \Phi_{S_{Z}}(x, \vec{r}),
$$

where $x$ is the center of mass coordinate for the two-bodies system, while the relative one is represented by $\vec{r}$. The index $S_{Z}$ is for the $z$-th component of the total spin $S$. 
The potential term $V(r)$ describes forces acting between two particles in the system. The absorptive part $\Gamma$ stands for the annihilation of the two-bodies system. For derivation of the effective Lagrangian and following evaluation of the annihilation cross section using it, see Ref. [6].

In the $\tilde{\chi}^{-} \tilde{\chi}^{-}$annihilation process with $S=0$, the potential $V(r)$ and absorptive part $\Gamma$ in Eq. (11) turn out to be

$$
V(r)=\frac{\alpha}{r}+\alpha_{2} c_{W}^{2} \frac{e^{-m_{Z} r}}{r}, \quad \Gamma=\frac{\pi \alpha_{2}^{2}}{2 m^{2}}
$$

where $\alpha$ is the fine structure constant, $\alpha_{2}=g_{2}^{2} / 4 \pi$ is for the $\mathrm{SU}(2)$ gauge coupling constant, $m_{Z}$ is the $Z$ boson mass, and $c_{W}=\cos \theta_{W}$ is for the weak mixing angle $\theta_{W}$. The first term in the potential comes from the exchange of photons, while the second one is from the exchange of $Z$ bosons. In the calculation of $\Gamma$, we consider only the final states of SM particles, and neglect their masses, since they are light enough compared to the wino-like neutralino we are discussing.

In both cases of the $\tilde{\chi}^{0} \tilde{\chi}^{-}$annihilation processes with $S=0$ and $1, V(r)$ is induced from the exchange of $W$ bosons, and both cases have the same form. On the other hand, the absorptive part $\Gamma$ is different each other. These are given by

$$
V(r)=-\alpha_{2} \frac{e^{-m_{W} r}}{r}, \quad \Gamma_{(S=0)}=\frac{1}{2} \frac{\pi \alpha_{2}^{2}}{m^{2}}, \quad \Gamma_{(S=1)}=\frac{25}{24} \frac{\pi \alpha_{2}^{2}}{m^{2}},
$$

where $m_{W}$ is the $W$ boson mass. The potential and absorptive terms in the $\tilde{\chi}^{-} \tilde{\chi}^{+}$ annihilation with $S=1$ are

$$
V(r)=-\frac{\alpha}{r}-\alpha_{2} c_{W}^{2} \frac{e^{-m_{Z} r}}{r}, \quad \Gamma=\frac{25}{24} \frac{\pi \alpha_{2}^{2}}{m^{2}} .
$$

The $\tilde{\chi}^{0} \tilde{\chi}^{0}$ two-bodies system is mixed with $\tilde{\chi}^{-} \tilde{\chi}^{+}$state with $S=0$, in which mixing occurs through a $W$ boson exchange. Thus, the potential and absorptive terms are written by $2 \times 2$ matrices as

$\mathbf{V}(r)=\left(\begin{array}{cc}2 \delta m-\frac{\alpha}{r}-\alpha_{2} c_{W}^{2} \frac{e^{-m_{Z^{r}}}}{r} & -\sqrt{2} \alpha_{2} \frac{e^{-m_{W} r}}{r} \\ -\sqrt{2} \alpha_{2} \frac{e^{-m_{W} r}}{r} & 0\end{array}\right), \boldsymbol{\Gamma}=\frac{\pi \alpha_{2}^{2}}{2 m^{2}}\left(\begin{array}{cc}3 & \sqrt{2} \\ \sqrt{2} & 2\end{array}\right)$.

Off-diagonal elements describe the transition between $\tilde{\chi}^{-} \tilde{\chi}^{+}$and $\tilde{\chi}^{0} \tilde{\chi}^{0}$ systems.

As seen in these potentials, all processes have attractive channels except for that of $\tilde{\chi}^{-} \tilde{\chi}^{-}$. The overlap between the wave functions of the incident particles are increased compared to the case without including the potentials, and it leads to enhancement of the annihilation cross sections. 
Once the two-bodies effective Lagrangian is obtained, annihilation cross sections including the non-perturbative effect can be calculated through the formula,

$$
\sigma v=c \Gamma|A|^{2}, \quad A \equiv \int d^{3} r e^{-i \vec{k} \cdot \vec{r}}\left(\frac{m v^{2}}{4}+\frac{\nabla^{2}}{m}\right) G(\vec{r}, \overrightarrow{0}),
$$

where $c=2$ for an annihilation of identical particles, otherwise $c=1$. The relative velocity between incoming particles is denoted by $v$. The Green function $G$ satisfies the equation of motion of the effective Lagrangian,

$$
\left[\frac{m v^{2}}{4}+\frac{\nabla^{2}}{m}-V(r)+2 i \Gamma \delta^{3}(\vec{r})\right] G\left(\vec{r}, \vec{r}^{\prime}\right)=\delta^{3}\left(\vec{r}-\vec{r}^{\prime}\right) .
$$

The boundary condition for the Green function is determined from following conditions. First, the Green function is analytic at any $\vec{r}$ and $\vec{r}^{\prime}$ except the point $\vec{r}=\vec{r}^{\prime}$. Second, only out-going waves survive at large $\left|\vec{r}-\vec{r}^{\prime}\right|$.

In $\tilde{\chi}^{0} \tilde{\chi}^{0}$ and $\tilde{\chi}^{-} \tilde{\chi}^{+}$annihilation processes with $S=0$, the Green function has a $2 \times 2$ matrix form. For this case, it has been found in Ref. [6, [7] that above formula is simply extended as

$$
\sigma_{i} v=c_{i} \sum_{j, j^{\prime}} \mathbf{A}_{i j} \boldsymbol{\Gamma}_{j j^{\prime}} \mathbf{A}_{i j^{\prime}}^{*}
$$

The factor $|A|^{2}$ in Eq. (6) is called the Sommerfeld enhancement factor, and it includes the non-perturbative effect due to the long-distance forces in $V(r)$. Note that if we neglect the non-perturbative effect, the enhancement factor becomes one and the annihilation cross section in Eq. (6) coincides with the result in a usual perturbative method.

\section{Effect of Sommerfeld enhancement on dark mat- ter abundance}

Now we evaluate the thermal relic abundance of the wino-like neutralino dark matter, including the non-perturbative effect. In the evaluation we have to include coannihilation processes in addition to the wino-like neutralino pair annihilation. We use the method developed in Ref. 9, 10, for the calculation of the relic abundance including coannihilation effects. Under reasonable assumptions, the relic density obeys the following Boltzmann equation,

$$
\frac{d Y}{d x}=-\frac{\left\langle\sigma_{\mathrm{eff}} v\right\rangle}{H x}\left(1-\frac{x}{3 g_{* s}} \frac{d g_{* s}}{d x}\right) s\left(Y^{2}-Y_{\mathrm{eq}}^{2}\right) .
$$


The yield of the dark matter, $Y$, is defined as $Y=n / s$, where $n$ is the sum of the number densities of $\tilde{\chi}^{0}, \tilde{\chi}^{-}$, and $\tilde{\chi}^{+}$. The variable, $x=m / T$, is the scaled inverse temperature of the universe. The equilibrium abundance, $Y_{\text {eq }}$, is given by

$$
Y_{\mathrm{eq}}=\frac{45}{2 \pi^{4}}\left(\frac{\pi}{8}\right)^{1 / 2} \frac{g_{\mathrm{eff}}}{g_{*}} x^{3 / 2} e^{-x}
$$

where $g_{\text {eff }}$ is the number of the effective degrees of freedom defined as

$$
g_{\text {eff }}(x)=2+4(1+\delta m / m)^{3 / 2} e^{-x \delta m / m} .
$$

The entropy density $s$ and the Hubble parameter $H$ are given by

$$
s=\frac{2 \pi^{2}}{45} g_{* s} \frac{m^{3}}{x^{3}}, \quad H=\left(\frac{g_{*}}{10}\right)^{1 / 2} \frac{\pi}{3 M_{\mathrm{Pl}}} \frac{m^{2}}{x^{2}},
$$

where $M_{\mathrm{Pl}}=2.4 \times 10^{18} \mathrm{GeV}$ is the reduced Planck mass. The relativistic degrees of freedom of the thermal bath, $g_{*}$ and $g_{* s}$, should be treated as a function of the temperature for deriving the correct dark matter abundance in our calculation.

The most important quantity to determine the abundance is the thermally averaged effective annihilation cross section $\left\langle\sigma_{\text {eff }} v\right\rangle$ in Eq. (9), defined as

$$
\begin{aligned}
\left\langle\sigma_{\mathrm{eff}} v\right\rangle & =\sum_{i, j}\left\langle\sigma_{i j} v\right\rangle \frac{4}{g_{\mathrm{eff}}^{2}(x)}\left(1+\Delta_{i}\right)^{3 / 2}\left(1+\Delta_{j}\right)^{3 / 2} \exp \left[-x\left(\Delta_{i}+\Delta_{j}\right)\right], \\
\left\langle\sigma_{i j} v\right\rangle & =\left(\frac{m}{4 \pi T}\right)^{3 / 2} \int 4 \pi v^{2} d v\left(\sigma_{i j} v\right) \exp \left(-\frac{m v^{2}}{4 T}\right)
\end{aligned}
$$

where $i, j=\tilde{\chi}^{0}, \tilde{\chi}^{-}$and $\tilde{\chi}^{+}, \Delta_{\tilde{\chi}^{-}}=\Delta_{\tilde{\chi}^{+}}=\delta m / m, \Delta_{\tilde{\chi}^{0}}=0$, and $\sigma_{i j}$ is the annihilation cross section between $i$ and $j$. In Fig. 1, the enhancement ratio of the averaged cross section, $\left\langle\sigma_{\text {eff }} v\right\rangle$, to that in the perturbative calculation is shown as a function of $m$ with fixed $m / T=20,200,2000$ (left figure) and as a function of $T$ with fixed $m=2.8 \mathrm{TeV}$ (right figure). For comparison, the cross section in a perturbative calculation is also shown as a dotted line in the right figure. Note that the perturbative cross section is constant in time. The little drop at $x \sim 10^{5}$ is due to the decoupling of $\tilde{\chi}^{ \pm}$. In the calculation of the cross section, we used the running gauge coupling constant at the $m$ and $m_{Z}$ in the absorptive terms and the potentials, respectively.

In these figures, large enhancement of the cross section is found due to the nonperturbative effect when $m$ is larger than $\sim 1 \mathrm{TeV}$. A significant enhancement is shown at $m \sim 2.4 \mathrm{GeV}$. This originates in the bound state composed of $\tilde{\chi}^{0} \tilde{\chi}^{0}$ and $\tilde{\chi}^{-} \tilde{\chi}^{+}$pairs [6, 7]. The enhancement by the non-perturbative effect is more significant for lower temperature. Since $\tilde{\chi}^{0}$ and $\tilde{\chi}^{-}$are more non-relativistic for lower 

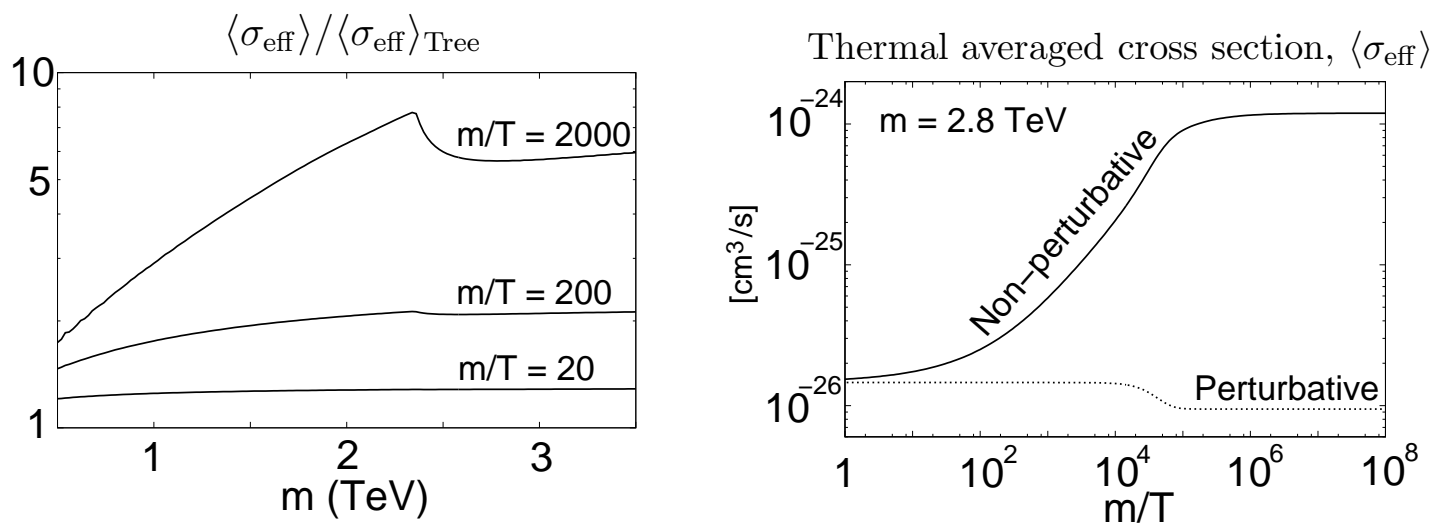

Figure 1: Mass dependence of averaged cross section, $\left\langle\sigma_{\text {eff }}\right\rangle$, normalized by the perturbative one, $\left\langle\sigma_{\text {eff }}\right\rangle_{\text {Tree }}$, for $m / T=20,200,2000$ (left figure), and temperature dependence with $m=2.8 \mathrm{TeV}$ (right figure). The perturbative result is also shown as a dotted line for comparison. Here, mass difference between $\tilde{\chi}^{0}$ and $\tilde{\chi}^{ \pm}$is $\delta m \simeq 0.17 \mathrm{GeV}$.

temperature, the long-range force acting between these particles strongly modifies their wave functions and alters the cross section significantly.

Since the averaged cross section depends on temperature in a non-trivial way as shown in Fig. 1, we should integrate the Boltzmann equation numerically. After calculating the present value of the yield, $Y_{0}$, by the integration, we obtain the dark matter mass fraction in the current universe through the relation $\Omega_{D M} h^{2}=$ $m s_{0} Y_{0} h^{2} / \rho_{c}$, where $\rho_{c}$ is the critical density, $\rho_{c}=1.05 \times 10^{-5} h^{2} \mathrm{GeVcm}^{-3}(h=$ $\left.0.73_{-0.03}^{+0.04}\right)$ [1], and $s_{0}$ is the entropy density of the present universe.

The result is shown in Fig. 2. In the left figure, the ratio of the yield with the enhancement to one without the enhancement (perturbative result) is shown as a function of temperature. The mass of the wino-like neutralino is assumed $m=$ 2.8 TeV. The enhancement of the cross section at the departure from the equilibrium decreases the abundance by $20-30 \%$, and it leads to quick deviation of the yield from the perturbative one after decoupling. Since the annihilation cross section is increased for lower temperature by the Sommerfeld enhancement, the sudden freezeout phenomenon on the yield does not occur and the yield continues to be reduced by the non-perturbative effect even for $x>100$ compared to the perturbative one. The resultant dark matter abundance is reduced by $50 \%$ compared to the perturbative result.

In the right figure, the relic abundance of the dark matter in the present universe is shown as a function of $m$ in terms of $\Omega h^{2}$. The allowed regions by the WMAP at 1 

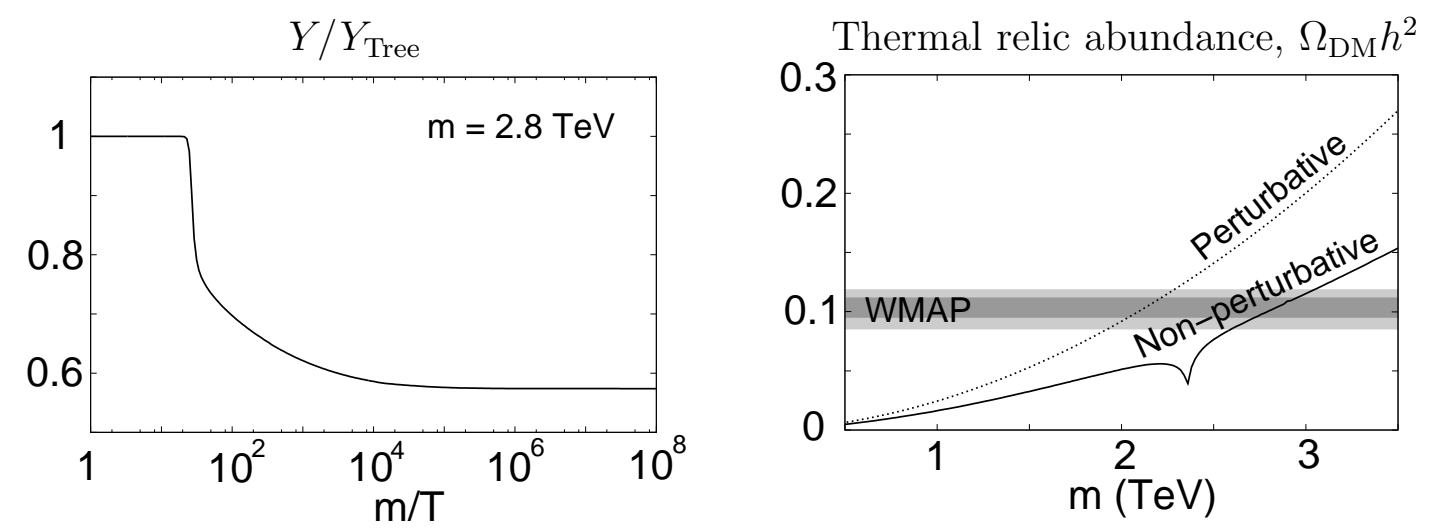

Figure 2: Ratio of yield with the non-perturbative effect to that in the perturbative calculation (left figure). Wino-like neutralino mass is fixed $2.8 \mathrm{TeV}$. Thermal relic abundance of the dark matter in the current universe as a function of wino-like neutralino mass (right figure). Allowed regions by the WMAP at $1(2) \sigma$ levels are also shown as the dark (light) shaded area.

and $2 \sigma$ are also shown as shaded areas in this figure. We found that the mass in the wino-like neutralino dark matter consistent with the observation is shifted by 600 $\mathrm{GeV}$ due to the non-perturbative effect and the wino-like neutralino mass consistent with WMAP results turns out to be $2.7 \mathrm{TeV} \lesssim m \lesssim 3.0 \mathrm{TeV}$.

\section{Summary and discussion}

In this letter, we have pointed out the thermal relic abundance of dark matter, which is $\mathrm{SU}(2)_{L}$ non-singlet and has a much larger mass than that of the weak gauge bosons, can be strongly reduced by the non-perturbative effect. We have investigated the non-perturbative effect on the relic abundance of wino-like neutralino as an example. Compared with the perturbative result, this effect reduces the abundance by about $50 \%$ and increases the mass of the wino-like neutralino dark matter consistent with the observation by about $600 \mathrm{GeV}$. As a result, the thermal relic abundance of the wino-like neutralino dark matter is consistent with observed abundances when $2.7 \mathrm{TeV} \lesssim m \lesssim 3.0 \mathrm{TeV}$

The non-perturbative effect can change relic abundances of other dark matter candidates with $\mathrm{SU}(2)_{L}$ charge and heavy mass, such as higgsino-like neutralino. The non-perturbative effect on the thermal relic abundance of higgsino-like neutralino is expected to be roughly $10 \%$, since winos are triplet under the $\mathrm{SU}(2)_{L}$ gauge group, 
while higgsinos are $\mathrm{SU}(2)_{L}$ doublet. Therefore, non-perturbative effect may change the abundance by $\mathrm{O}(10 \%)$ for other $\mathrm{SU}(2)_{L}$ doublet candidates for the dark matter. A detailed analysis of this subject is studied elsewhere.

The Sommerfeld enhancement occurs reasonably for particles with electric charge. In fact the non-perturbative effect through photon exchanges for charged particle annihilation can change the abundance by about $10 \%$. Therefore, one may think that the relic abundance of dark matter will be changed in the stau coannihilation region or in the case that gravitino is the lightest supersymmetric particle and it is produced through decay of stau. However, the non-perturbative effect for stau does not change the dark matter abundance. In stau annihilation, $\sigma\left(\tilde{\tau}^{+} \tilde{\tau}^{-}\right) \simeq \sigma\left(\tilde{\tau}^{+} \tilde{\tau}^{+}\right)$and the Sommerfeld enhancement is positive (negative) for the former (latter) process. Hence, the enhancement is almost canceled and the change of the abundance is $1 \%$ at most.

Kaluza-Klein $(\mathrm{KK})$ right-handed leptons $\left(E^{(1)}\right)$ in UED models are highly degenerate with the lightest $\mathrm{KK}$ particle in mass and $\sigma\left(E^{(1)} \bar{E}^{(1)}\right)>\sigma\left(E^{(1)} E^{(1)}\right)$ [3] 12 . Hence, the enhancement could be expected to change the abundance of the KK dark matter. The change of the abundance is within $4 \%$ since $\sigma\left(E^{(1)} \bar{E}^{(1)}\right)$ contributes to the effective annihilation cross section by $40 \%$ at most.

Finally, we comment on the non-perturbative effect of colored particles, such as gluino. The enhancement for colored particles are very effective [13]. However, it may be very complicated due to the existence of the QCD phase transition, which is discussed in Ref. 14. Furthermore, colored particles can not be candidates for the dark matter and are not expected to be degenerate with a dark matter particle in mass due to large radiative corrections by the strong interaction. Hence, this subject is beyond the scope of this letter.

\section{Acknowledgments}

This work is supported in part by the Grant-in-Aid for Science Research, Ministry of Education, Science and Culture, Japan (No.1803422 and 15540255 for JH, 16081211 for SM and 18840011 for MS). Also, the work of MN is supported in part by JSPS. 


\section{References}

[1] C. L. Bennett et al., Astrophys. J. Suppl. 148, 1 (2003); D. N. Spergel et al. [WMAP Collaboration], Astrophys. J. Suppl. 148, 175 (2003); D. N. Spergel et al., arXiv:astro-ph/0603449.

[2] T. Appelquist, H. C. Cheng and B. A. Dobrescu, Phys. Rev. D 64, 035002 (2001).

[3] G. Servant and T. M. P. Tait, Nucl. Phys. B 650, 391 (2003);

[4] For reviews, see for instance, G. Jungman, M. Kamionkowski and K. Griest, Phys. Rept. 267, 195 (1996); L. Bergstrom, Rept. Prog. Phys. 63, 793 (2000); C. Munoz, Int. J. Mod. Phys. A 19, 3093 (2004); G. Bertone, D. Hooper and J. Silk, Phys. Rept. 405, 279 (2005).

[5] M. Kakizaki, S. Matsumoto and M. Senami, Phys. Rev. D 74, 023504 (2006).

[6] J. Hisano, S. Matsumoto, M. M. Nojiri and O. Saito, Phys. Rev. D 71, 063528 (2005).

[7] J. Hisano, S. Matsumoto and M. M. Nojiri, Phys. Rev. Lett. 92, 031303 (2004).

[8] J. Hisano, S. Matsumoto, O. Saito and M. Senami, Phys. Rev. D 73, 055004 (2006).

[9] K. Griest and D. Seckel, Phys. Rev. D 43, 3191 (1991).

[10] P. Gondolo and G. Gelmini, Nucl. Phys. B 360, 145 (1991).

[11] W. M. Yao et al. [Particle Data Group], J. Phys. G 33,1 (2006).

[12] M. Kakizaki, S. Matsumoto, Y. Sato and M. Senami, Nucl. Phys. B 735, 84 (2006).

[13] H. Baer, K. m. Cheung and J. F. Gunion, Phys. Rev. D 59, 075002 (1999).

[14] A. Arvanitaki, C. Davis, P. W. Graham, A. Pierce and J. G. Wacker, Phys. Rev. D 72, 075011 (2005). 